\title{
NAG1-01092
}

\section{A Discontinuous Galerkin Method for Parabolic Problems with Modified $h p$-Finite Element Approximation Technique}

\author{
Hideaki Kaneko* \\ Department of Mathematics and Statistics \\ Old Dominion University \\ Norfolk, Virginia 23529-0077 \\ Kim S. Bey \\ Thermal Structures Branch \\ Structure Division \\ NASA Langley Research Center \\ Hampton, VA 23681 \\ Gene J. W. Hou ${ }^{\dagger}$ \\ Department of Mechanical Engineering \\ Old Dominion University \\ Norfolk, Virginia 23529
}




\title{
A Discontinuous Galerkin Method for Parabolic Problems with Modified $h p$-Finite Approximation Technique
}

\author{
H. Kaneko, G. J. W. Hou, and K. S. Bey
}

\begin{abstract}
A recent paper [1] is generalized to a case where the spatial region is taken in $R^{3}$. The region is assumed to be a thin body, such as a panel on the wing or fuselage of an aerospace vehicle. The traditional $h$ - as well as $h p$-finite element methods are applied to the surface defined in the $x-y$ variables, while, through the thickness, the technique of the $p$-element is employed. Time and spatial discretization scheme developed in [1], based upon an assumption of certain weak singularity of $\left\|u_{t}\right\|_{2}$, is used to derive an optimal a priori error estimate for the current method.
\end{abstract}

Key words: Discontinuous Galerkin Method, Parabolic Equations, Modified $h p$-Finite Element Method.

\section{Introduction}

In this paper, the discontinuous Galerkin method is applied to the following standard model problem of parabolic type:

Find $u$ such that

$$
\begin{array}{ll}
u_{t}(x, t)-\Delta u(x, t)=f(x, t), & x \in \Omega, t \in R^{+}, \\
u(x, t)=0, & x \in \partial \Omega, t \in R^{+}, \\
u(x, 0)=u_{0}(x), & x \in \Omega,
\end{array}
$$

where $\Omega$ is a closed and bounded set in $R^{3}$ with boundary $\partial \Omega, R^{+}=(0, \infty), \Delta u=$ $\partial^{2} u / \partial x^{2}+\partial^{2} / \partial y^{2}+\partial^{2} u / \partial z^{2}, u_{t}=\partial u / \partial t$, and the functions $f$ and $u_{0}$ are given data.

*This author is supported by NASA- Grant NAG-1-01092

This author is supported by NASA- Grant NAG-1-2300 
The discontinuous Galerkin method is a robust finite element method that can deliver high-order numerical approximation using unstructured grids. In this paper, region $\Omega$ is assumed to be a thin body in $R^{3}$, such as a panel on the wing or fuselage of an aerospace vehicle. The traditional $h$ - as well as $h p$-finite element approximations are used in the $x-y$ variables, whereas, the $p$-finite element method developed, e.g., in [5],[15], is used in the $z$ variable which describes the region through the thickness. The application of the $p$-finite element method through the thickness of thin structure, as compared to applying the $h$ - or $h p$-finite element discretization to all coordinate directions, enables us to avoid structuring elements in $R^{3}$ that are too thin to satisfy the required quasi-uniformity condition (e.g. see [7]) that is necessary to deliver stable numerical approximation. We are coining the term 'modified $h p$ '-finite element method, as it differs from the traditional $h p$-finite element method which uses $h$ - and $p$-finite elements on the same domain where the $h$-finite element method provides a refinement of the region and the $p$-finite clement provides an enrichment. In Section 2, approximation power of the modified $h p$-finite element method will be investigated. In Section 3, the discontinuous Galerkin method with the modified $h p$-finite element approximation technique is established. Discontinuity is in time variable and time discretization is based upon the degree of singularity of $\left\|u_{t}\right\|_{2}$. The traditional $h$-finite element method is employed in time. The convergence analysis given in [9] will be used. The reader is also reminded of recently published important paper [16] by Schötzau and Schwab in which various time discretization techniques are discussed. For instance, an exponential convergence rate in time of $p$-finite element method is obtained in [16] despite the presence of singularity in the transient phase of the solution. Time discretization used there is geometric. Schötzau and Schwab's result extends the results in [3] and [4] in which no exponential convergence rate is reported. Also they discuss the $h$-finite element technique in time using a class of radical mesh and obtain the algebraic convergence rate which is optimal. The radical mesh was chosen by analyzing the incompatibility between initial and boundary data. The present authors [1] established a similar time discretization technique for the discontinuous Galerkin finite element method, $h$-version in time, which was based upon the singularity of $\left\|u_{t}\right\|_{2}$. Using 
this analysis, it is shown in [1] that the optimal algebraic convergence rate in time of the discontinuous Galerkin method can be obtained under more dispersed, therefore more computationally stable radical mesh than the mesh used in [16].

\section{Approximation Power of Modified $h p$ Elements}

Let $\omega \subseteq R^{2}$ and $\Gamma \subseteq R$ be convex regions. For simplicity, it is assumed that $\Gamma \equiv\left[-\frac{d}{2}, \frac{d}{2}\right]$ where $d=|\Gamma|$. For simplicity, the thickness, $\mathrm{d}$, is assumed constant over the domain. The Sobolev space of order $k$ defined on $\omega \times \Gamma$ is denoted by $H^{k}(\omega \times \Gamma)$ with the norm

$$
\|u\|_{k, \omega \times \Gamma}^{2}=\sum_{0 \leq|\alpha| \leq k}\left\|D^{\alpha} u\right\|_{2}^{2}
$$

where for each multi-integer $\alpha=\left(\alpha_{1}, \alpha_{2}, \alpha_{3}\right)$, we have let $|\alpha|=\alpha_{1}+\alpha_{2}+\alpha_{3}$ and

$$
D^{\alpha}=\frac{\partial^{|\alpha|}}{\partial x_{1}^{\alpha_{1}} \partial x_{2}^{\alpha_{2}} \partial x_{3}^{\alpha_{3}}} .
$$

We note that the Sobolev norm reduces to the usual $L_{2}$ norm when $k=0$. In this section, a best possible error estimate is derived for approximating an element in $H^{k}(\omega \times \Gamma)$ by the finite element function spaces. Let $K_{\xi, \eta}$ denote the master triangular element defined by

$$
\begin{array}{rlc}
K_{\xi, \eta}=\left\{(\xi, \eta) \in R^{2}:\right. & 0 \leq \eta \leq(1+\xi) \sqrt{3} & -1 \leq \xi \leq 0 \text { or } \\
& 0 \leq \eta \leq(1-\xi) \sqrt{3} & 0 \leq \xi \leq 1\} .
\end{array}
$$

Let $S^{p}\left(K_{\xi, \eta}\right)$ denote the space of polynomials of degree $\leq p$ on $K_{\xi, \eta}$, -i.e.,

$$
S^{p}\left(K_{\xi, \eta}\right)=\operatorname{span}\left\{\xi^{i} \eta^{j}: i, j=0,1, \ldots, p ; i+j \leq p\right\}
$$

First, the shape functions for the master element $K_{\xi, \eta}$ are formed. To accomplish this, the barycentric coordinates are introduced via

$$
\lambda_{1}=(1-\xi-\eta / \sqrt{3}) / 2, \quad \lambda_{2}=(1+\xi-\eta / \sqrt{3}) / 2, \quad \lambda_{3}=\eta / \sqrt{3}
$$

$\lambda_{i}$ 's form a partition of unity and $\lambda_{i}$ is identically equal to one at a vertex of $K_{\xi, \eta}$ and vanishes on the opposite side of $K_{\xi, \eta}$. The hierarchical shape functions on $K_{\xi, \eta}$ consists 
of internal as well as external functions. The normalized antiderivatives of the Legendre polynomials are defined by

$$
\bar{\psi}_{i}(\zeta)=\sqrt{\frac{2 i+1}{2}} \int_{-1}^{\zeta} P_{i}(t) d t, \quad i=1,2,3, \ldots
$$

Now, the external shape functions consist of 3 nodal shape functions

$$
N_{i}(\xi, \eta)=\lambda_{i}, \quad i=1,2,3
$$

and $3(p-1)$ side shape functions $N_{i}^{[j]}(\xi, \eta), i=1, \ldots, p-1, j=1,2,3$. The index $j$ indicates one of three sides of $K_{\xi, \eta}$. Noting that $\bar{\psi}_{i}( \pm 1)=0$,

$$
\bar{\psi}_{i}(\eta)=\frac{1}{4}\left(1-\eta^{2}\right) \varphi_{i}(\eta), \quad i=1,2,3, \ldots
$$

where $\varphi_{i}(\eta)$ is a polynomial of degree $i-1$. For instance, $\varphi_{1}(\eta)=-\sqrt{6}, \varphi_{2}(\eta)=-\sqrt{10} \eta$ and $\varphi_{3}(\eta)=\frac{\sqrt{14}}{4}\left(1-5 \eta^{2}\right)$, etc. The side shape functions are constructed as follows:

$$
\begin{aligned}
& N_{i}^{[1]}(\xi, \eta)=\lambda_{2} \lambda_{3} \varphi_{i}\left(\lambda_{3}-\lambda_{2}\right) \\
& N_{i}^{[2]}(\xi, \eta)=\lambda_{3} \lambda_{1} \varphi_{i}\left(\lambda_{1}-\lambda_{3}\right), \quad i=1, \ldots, p-1, \\
& N_{i}^{[3]}(\xi, \eta)=\lambda_{1} \lambda_{2} \varphi_{i}\left(\lambda_{2}-\lambda_{1}\right) .
\end{aligned}
$$

From (2.4) and (2.5), there are $3+3(p-1)$ shape functions. As $\operatorname{dim}\left(S^{p}\left(K_{\xi, \eta}\right)\right)=\frac{(p+1)(p+2)}{2}$, the remaining $\frac{(p-1)(p-2)}{2}$ basis elements are constructed in terms of internal shape functions. Clearly, nontrivial internal shape functions on $K_{\xi, \eta}$ exists only if $p \geq 3$. For $p=3$, the bubble function on $K_{\xi, \eta}$ below serves as the internal function;

$$
b_{K_{\xi, \eta}}(\xi, \eta)=\lambda_{1} \lambda_{2} \lambda_{3}=\frac{\eta}{4 \sqrt{3}}\left(\left(1-\frac{\eta}{\sqrt{3}}\right)^{2}-\xi^{2}\right) .
$$

Moreover, the collection $I^{p}\left(K_{\xi, \eta}\right)$ of higher-order internal shape functions can be constructed from

$$
I^{p}\left(K_{\xi, \eta}\right)=\left\{b_{K_{\xi, \eta}} v: v \in S^{p-3}\left(K_{\xi, \eta}\right\}=\left\{b_{K_{\xi, \eta}}\right\} \otimes S^{p-3}\left(K_{\xi, \eta}\right), \quad p \geq 3 .\right.
$$

Let $T_{h}, h>0$, be a triangulation of $\omega$. let $x=Q_{x}^{l}\left(L_{1}, L_{2}, L_{3}\right)$ and $y=Q_{y}^{l}\left(L_{1}, L_{2}, L_{3}\right)$ be the element mappings of the standard triangle $K_{\xi, \eta}$ to the $l$ th triangular element $K^{l} \in T_{h}$, e.g., the linear mapping onto $K^{l}$ with vertices $\left\{\left(x_{i}^{l}, y_{i}^{l}\right)\right\}_{i=1}^{3}$,

$$
Q_{x}^{l}\left(L_{1}, L_{2}, L_{3}\right)=\sum_{i=1}^{3} x_{i}^{l} L_{i}, \quad Q_{y}^{l}\left(L_{1}, L_{2}, L_{3}\right)=\sum_{i=1}^{3} y_{i}^{l} L_{i} .
$$


The space of all polynomials of degree $\leq p$ on $K^{l}$ is denoted by $S^{p}\left(K^{l}\right)$ and its basis can be formed from the shape functions of $S^{p}\left(K_{\xi, \eta}\right)$ described above by transforming them under $Q_{x}^{l}$ and $Q_{y}^{l}$. The finite element space $S^{p, l}\left(\omega, T_{h}\right)$ is now defined. For $\omega, p \geq 0$ and $k \geq 0$,

$$
S^{p, k}\left(\omega, T_{h}\right)=\left\{u \in H^{k}(\omega):\left.u\right|_{K} \in S^{p}(K), K \in T_{h}\right\} .
$$

Assume that a triangulation $\left\{T_{h}\right\}, h>0$, of $\omega$ consists of $\left\{K_{h}^{l}\right\}_{l=1}^{M(h)}$ and that $h_{l}=$ $\operatorname{diam}\left(K_{h}^{l}\right)$, for $l=1, \ldots, M(h)$.

In the $z$-variable for through the thickness approximation, the local variable $\tau$ is defined in the reference element $[-1,1]$ and $\Gamma$ is mapped onto the reference element by $Q_{z}$, i.e.,

$$
\Gamma=Q_{z}([-1,1]), \quad z=Q_{z}(\tau)
$$

Clearly, $Q_{z}$ is a linear function defined by

$$
z=Q_{z}(\tau)=\frac{1}{2}(1-\tau)\left(-\frac{d}{2}\right)+\frac{1}{2}(1+\tau) \frac{d}{2}, \quad \tau \in[-1,1]
$$

The Jacobian of $Q_{z}$ is constant

$$
\frac{d z}{d \tau}=\frac{d}{2}
$$

In this paper, the basis functions of $P_{p}([-1,1])$ are taken to be the one-dimensional hierarchical shape functions. See [15] for a complete discussion of the basis elements used in the $p$ and $h p$-finite element methods.

For example, in approximating an element in $H^{l}[-1,1]$, with $l=0, \psi_{i}(\tau)=P_{i-1}(\tau)$, $1 \leq i \leq p+1$, where $P_{i-1}$ is the Legendre polynomial of degree $i-1$, form the hierarchical basis functions. With $l=1$, the external $\left(\psi_{1}\right.$ and $\left.\psi_{2}\right)$ and internal $\left(\psi_{i}, i \geq 3\right)$ shape functions are defined by

$$
\begin{aligned}
& \psi_{1}(\tau)=\frac{1-\tau}{2}, \quad \psi_{2}(\tau)=\frac{1+\tau}{2} \\
& \psi_{i}(\tau)=\left(\frac{2 i-3}{2}\right)^{1 / 2} \int_{-1}^{\tau} P_{i-2}(t) d t, \quad 3 \leq i \leq p+1
\end{aligned}
$$

Note that $\psi_{i}$ 's form an orthogonal family with respect to the energy inner product $(\cdot, \cdot)_{E}$,

$$
\left(\psi_{i}, \psi_{j}\right)_{E} \equiv \int_{-1}^{1} \psi_{i}^{\prime}(t) \psi_{j}^{\prime}(t) d t=\int_{-1}^{1} P_{i}(t) P_{j}(t) d t=\delta_{i j}
$$


Also note that the internal shape functions satisfy

$$
\psi_{i}( \pm 1)=0, \quad \text { for } 3 \leq i \leq p+1 \text {. }
$$

For the case $l=2$ and $p \geq 3$, the four nodal shape functions and the remaining $p-3$ internal shape functions given by

$$
\begin{aligned}
& \psi_{1}(\tau)=\frac{1}{4}(1-\tau)^{2}(1+\tau), \quad \psi_{2}(\tau)=\frac{1}{4}(1-\tau)^{2}(2+\tau) \\
& \psi_{3}(\tau)=-\frac{1}{4}(1+\tau)^{2}(1-\tau), \quad \psi_{4}(\tau)=\frac{1}{4}(1+\tau)^{2}(2-\tau) \\
& \psi_{i}(\tau)=\left(\frac{2 i-5}{2}\right)^{1 / 2} \int_{-1}^{\tau}(\tau-\eta) P_{i-3}(\eta) d \eta, \quad i=5, \ldots, p+1
\end{aligned}
$$

In this case, the internal shape functions satisfy

$$
\frac{d^{j} \psi_{i}}{d \tau^{j}}( \pm 1)=0, \quad \text { for } 5 \leq i \leq p+1 \text { and } j=0,1
$$

The nodal basis functions, $\psi_{i}, i=1,2,3,4$, in (2.9) also satisfy three of the four conditions in (2.10). For example, using the shape functions in (2.8), any element $u \in L_{2}[-1,1]$ can be approximated by $u_{p} \in P_{p}([-1,1])$, in the form

$$
u_{p}(\tau)=\frac{1-\tau}{2} u(-1)+\frac{1+\tau}{2} u(1)+\sum_{i=3}^{p+1} a_{i} \psi_{i}(\tau)
$$

For approximating the solutions of parabolic problems with the homogeneous Dirichlet boundary condition, the first two terms will be dropped, as $u(-1)=u(1)=0$. A sequence of triangulations $\left\{T_{h}\right\}_{h>0}$ is called the quasiuniform mesh if

$$
\frac{h}{\operatorname{diam}(K)} \leq \gamma, \quad \text { for all } h>0
$$

with $h=\max _{K \in T_{h}} \operatorname{diam}(K)$, and for some $\gamma>0 . P_{p}(\Gamma)$ denotes the space of all polynomials of degree $\leq p$ defined on $\Gamma$. The following is proved by Babuška, Szabo and Katz in [5]. See also [6] by Babuška and Suri on a related discussion. Here $\Omega_{0}$ denotes a bounded polygonal domain in $R^{2}$.

Theorem 2.1 Let $u \in H^{k}\left(\Omega_{0}\right)$. Then there exists a sequence $z_{p} \in P_{p}\left(\Omega_{0}\right), p=1,2, \ldots$, such that, for any $0 \leq l \leq k$,

$$
\left\|u-z_{p}\right\|_{l, \Omega_{0}} \leq C p^{-(k-l)}\|u\|_{k, \Omega_{0}}
$$

where $C$ is independent of $u$ and $p$. 
The parameters $k$ and $l$ are not necessarily integral. Their proof relies heavily on the approximation power of the trigonometric polynomials.

With $l=0$ in Theorem 2.1 and using the usual duality argument, the results in Theorem 2.1 are further improved by Babuška and Suri in [6] (theorem 2.9), (see also a series of papers by Gui and Babuška [13]), to the $h p$-finite element setting as follows:

Theorem 2.2 Let $T_{h}$ be a quasiuniform partition of $\Omega_{0}$. Then for $k \geq 1, u \in H^{k}\left(\Omega_{0}\right)$,

$$
\inf _{v \in S^{p, k}\left(\omega, T_{h}\right)}\|u-v\|_{L_{2}\left(\Omega_{0}\right)} \leq C h^{\nu} p^{-k}\|u\|_{H^{k}\left(\Omega_{0}\right)}
$$

where $\nu=\min (k, p+1)$.

The corresponding error estimate in the $\|\cdot\|_{H^{k}\left(\Omega_{0}\right)}$ is also available in [6].

$\mathrm{h}$-version in the $x-y$ surface variables: First, the $\mathrm{h}$-finite element approximation is considered in the $x-y$ variables. Let $z=s(\tau)=\frac{d}{2} \tau$ be the linear transformation of $[-1,1]$ onto $\Gamma$. Now consider the problem of approximating a function $u \in H^{k}(\omega \times \Gamma)$ by a function from the tensor product space $S_{h}^{r}(\omega) \otimes P_{p}(\Gamma)$, where

$$
S_{h}^{r}(\omega)=S_{h}^{r}\left(\omega, T_{h}\right)=\left\{u \in L_{2}(\omega): u \mid K \in S^{r}(K), K \in T_{h}\right\}
$$

For error analysis of $h$-version of the finite element method, the space $S^{p, k}\left(\omega, T_{h}\right)$ defined in (2.7) is not necessary, and the space $S_{h}^{r}(\omega)$ of lower dimension can be used to attain the optimal convergence rate. Let $P_{h}^{r}: H^{2}(\omega) \rightarrow S_{h}^{r}(\omega)$ denote the interpolation projection defined by

$$
\left(P_{h}^{r} u\right)(x, y)=\sum_{i=1}^{r} u\left(x_{i}^{l}, y_{i}^{l}\right) \varphi_{i}^{l}(x, y), \quad \text { for all }(x, y) \in K^{l} \text { and } u \in H^{k}(\omega)
$$

where $T_{h}$ is a triangulation of $\omega$ with $K^{l} \in T_{h}$ and $\left\{\left(x_{i}^{l}, y_{i}^{l}\right)\right\}_{i=1}^{r}$ is a set of nodes on $K^{l}$ with $\varphi_{i}^{l}\left(x_{j}^{l}, y_{j}^{l}\right)=\delta_{i j}$. Also, denote by $Q_{p}: H^{k}(\Gamma) \rightarrow P_{p}(\Gamma)$ a projection defined by

$$
\left(Q_{p} u\right)(z)=\sum_{i=1}^{p+1} a_{i} \Psi_{i}(z), \quad \text { for all } z \in \Gamma
$$


where $\Psi_{i}(z)=\psi_{i}\left(s^{-1}(z)\right)$ for each $i \geq 1$ where $\psi_{i}$ are defined, e.g., in (2.8) or (2.9). Recall that for $k=1$, the constants $a_{1}$ and $a_{2}$ are known in case of parabolic problems with Dirichlet condition, and it is assumed that $a_{i}, i \geq 3$, in (2.15) are determined by

$$
\int_{\Gamma}\left|u(z)-\sum_{j=3}^{p+1} a_{j} \Psi_{j}(z)\right|^{2} d z=\min _{b_{j} \in R} \int_{\Gamma}\left|u(z)-\sum_{j=3}^{p+1} b_{j} \Psi_{j}(z)\right|^{2} d z
$$

From approximation theory [17],

$$
\left\|I-P_{h}^{r}\right\|_{L_{2}(\Omega)}=O\left(h^{r}\right)
$$

Also $Q_{p}: L_{2}(\Gamma) \rightarrow P_{p}(\Gamma)$, from being the orthogonal projection in the sense described in (2.16) and from Theorem 2.1 that

$$
\left\|I-Q_{p}\right\|_{L_{2}(\Gamma)}=O\left(p^{-k}\right) .
$$

Let

$$
\left\|P_{h}^{r} \otimes Q_{p}\right\|_{2} \equiv\left\|P_{h}^{r} \otimes Q_{p}\right\|_{L_{2}(\omega \times \Gamma)} \equiv \sup _{\|u\|_{2}=1}\left\|\left(P_{h}^{r} \otimes Q_{p}\right) u\right\|_{L_{2}(\omega \times \Gamma)}
$$

For $u \in L_{2}(\omega \times \Gamma)$,

$$
\left(P_{h}^{r} \otimes I\right) u(x, y, z)=\sum_{l} \sum_{i=1}^{r} u\left(x_{i}^{l}, y_{i}^{l}, z\right) \varphi_{i}^{l}(x, y)
$$

and

$$
\left(P_{h}^{r} \otimes Q_{p}\right) u(x, y, z)=\sum_{j=1}^{p+1}\left\{\sum_{l} \sum_{i=1}^{r} \varphi_{i}^{l}(x, y)\right\} a_{j} \Psi_{j}(z),
$$

where $a_{j}$ depends upon $u$ and obtained according to (2.16). First, approximation order under $L_{2}$ operator norm of $P_{h}^{r} \otimes Q_{p}$ for $P_{h}^{r} \otimes I$ is established.

Lemma 2.3 For $P_{h}^{r}: H^{k}(\omega) \rightarrow S_{h}^{r}(\omega), 0 \leq r \leq k$, and $Q_{p}: H^{k}(\Gamma) \rightarrow P_{p}(\Gamma)$ defined respectively in (2.14) and (2.15),

$$
\left\|P_{h}^{r} \otimes I-P_{h}^{r} \otimes Q_{p}\right\|_{2} \leq C p^{-k}
$$

where $C$ is independent of $p$. 


\section{Proof:}

$$
\begin{aligned}
& \left\|P_{h}^{r} \otimes I-P_{h}^{r} \otimes Q_{p}\right\|_{2} \equiv \sup _{\|u\|_{2}=1}\left\|\left(P_{h}^{r} \otimes I-P_{h}^{r} \otimes Q_{p}\right) u\right\|_{2} \\
& =\sup _{\|u\|_{2}=1}\left\{\sum_{l} \int_{K^{l}} \int_{\Gamma} \mid \sum_{i=1}^{r} u\left(x_{i}^{l}, y_{i}^{l}, z\right) \varphi_{i}^{l}(x, y)-\right. \\
& \left.\sum_{j=1}^{p+1} \sum_{i=1}^{r} \varphi_{i}^{l}(x, y) a_{j} \Psi_{j}(z) \mid d z d x d y\right\}^{1 / 2} \\
& \leq \sup _{\|u\|_{2}=1}\left\{\sum_{l} \int_{K^{l}} \int_{\Gamma}\left|\sum_{i=1}^{r} \varphi_{i}^{l}(x, y)\left(u\left(x_{i}^{l}, y_{i}^{l}, z\right)-\sum_{j=1}^{p+1} a_{j} \Psi_{j}(z)\right)\right|^{2} d z d x d y\right\}^{1 / 2} \\
& \leq \sup _{\|u\|_{2}=1} \sum_{i=1}^{r}\left\{\sum_{l} \int_{K^{l}} \int_{\Gamma}\left|\varphi_{i}^{l}(x, y)\right|^{2}\left|u\left(x_{i}^{l}, y_{i}^{l}, z\right)-\sum_{j=1}^{p+1} a_{j} \Psi_{j}(z)\right|^{2} d z d x d y\right\}^{1 / 2} \\
& \text { by Minkowski inequality } \\
& \leq \sup _{\|u\|_{2}=1}\left\{r \max _{1 \leq i \leq r}\left\{\sum_{l} \int_{K^{2}}\left|f_{i}^{l}(x, y)\right|^{2}\left|u\left(x_{i}^{l}, y_{i}^{l}, z\right)-\sum_{j=1}^{p+1} a_{j} \Psi_{j}(z)\right|^{2} d z d x d y\right\}^{1 / 2}\right. \\
& \leq C \sup _{\|u\|_{2}=1} \max _{1 \leq i \leq r}\left\{\int_{\Gamma}\left|u\left(x_{i}^{l}, y_{i}^{l}, z\right)-\sum_{j=1}^{p+1} a_{j} \Psi_{j}(z)\right|^{2} d z\right\}^{1 / 2} \\
& \text { where } M \equiv \sum_{l} \int_{K^{l}}\left|\varphi_{i}^{l}(x, y)\right|^{2} d x d y \\
& \leq C p^{-k}, \quad \text { by Theorem } 2.1 \text {. }
\end{aligned}
$$

Similarly, the following lemma will be useful.

Lemma 2.4 Let $P_{h}^{r}: H^{k}(\omega) \rightarrow S_{h}^{r}$, with $0 \leq r \leq k$ and $Q_{p}: H^{k}(\Gamma) \rightarrow P_{p}(\Gamma)$. Then

$$
\left\|I \otimes Q_{p}-P_{h}^{r} \otimes Q_{p}\right\|_{2} \leq C h^{r}
$$

where $C$ is independent of $r$. 


\section{Proof:}

$$
\begin{aligned}
& \left\|I \otimes Q_{p}-P_{h}^{r} \otimes Q_{p}\right\|_{2} \equiv \sup _{\|u\|_{2}=1}\left\|\left(I \otimes Q_{p}-P_{h}^{r} \otimes Q_{p}\right) u\right\|_{2} \\
& =\sup _{\|u\|_{2}=1}\left\{\sum_{K^{\prime} \in T_{h}} \int_{K^{l}} \int_{\Gamma} \mid \sum_{j=1}^{p+1} \alpha_{j}(x, y) \Psi_{j}(z)-\right. \\
& \left.\left.\sum_{j=1}^{p+1}\left(\sum_{i=1}^{r} \alpha_{j}\left(x_{i}^{l}, y_{i}^{l}\right) \varphi_{i}(x, y)\right) \Psi_{j}(z)\right|^{2} d z d x d y\right\}^{1 / 2} \\
& \text { where } \sum_{j=1}^{p+1} \alpha_{j}(x, y) \Psi_{j}(z) \text { is the best } L_{2}(\Gamma) \text { approximation of } u(x, y, \cdot) \\
& \leq \sup _{\|u\|_{2}=1}\left\{\sum_{K^{l} \in T_{h}} \int_{K^{l}} \int_{\Gamma}\left|\sum_{j=1}^{p+1}\left\{\alpha_{j}(x, y)-\sum_{i=1}^{r} \alpha_{j}\left(x_{i}^{l}, y_{i}^{l}\right) \varphi_{i}(x, y)\right\} \Psi_{j}(z)\right|^{2} d z d x d y\right\}^{1 / 2} \\
& \leq \sup _{\|u\|_{2}=1} \sum_{j=1}^{p+1}\left\{\sum_{K^{l} \in T_{h}} \int_{K^{l}} \int_{\Gamma}\left|\alpha_{j}(x, y)-\sum_{i=1}^{r} \alpha_{j}\left(x_{i}^{l}, y_{i}^{l}\right) \varphi_{i}(x, y)\right|^{2}\left|\Psi_{j}(z)\right|^{2} d z d x d y\right\}^{1 / 2} \\
& \leq C\left\{\sum_{K^{l} \in T_{h}} \int_{K^{l}}\left|\alpha_{j}(x, y)-\sum_{i=1}^{r} \alpha_{j}\left(x_{i}^{l}, y_{i}^{l}\right) \varphi_{i}(x, y)\right|^{2} \mid d x d y\right\}^{1 / 2} \\
& \leq C h^{r}, \quad \text { provided that } \alpha_{j} \in H^{r}(\omega),
\end{aligned}
$$

where the last inequality follows from a well known result of the approximation power of piecewise polynomials [17].

Using Lemmas 2.1 and 2.2, we obtain the following theorem which provides an error estimate for approximating an element in $H^{k}(\omega \times \Gamma)$ by elements from $S_{h}^{r}(\omega) \otimes P_{p}(\Gamma)$. The result will be used in the next section when the formulation of error estimate of the modified $h-p$ discontinuous Galerkin finite element method for approximating the solution of the parabolic problem (1.1) is established.

Theorem 2.5 Let $u \in H^{k}(\omega \times \Gamma)$. Then there exists $u^{*} \in S_{h}^{r}(\omega) \otimes P_{p}(\Gamma)$ such that for $0 \leq r \leq k, p>0$,

$$
\left\|u-u^{*}\right\|_{L_{2}(\omega \times \Gamma)}=O\left(h^{r}+p^{-k}\right) .
$$

Proof: Define $u^{*}=\left(P_{h}^{r} \otimes Q_{p}\right) u$. Then, using Lemmas 2.3 and 2.4

$$
\begin{aligned}
\left\|u-u^{*}\right\|_{2} & =\left\|u-\left(P_{h}^{r} \otimes Q_{p}\right) u\right\|_{2} \\
& =\left\|u-\left(I \otimes Q_{p}\right) u+\left(I \otimes Q_{p}\right) u-\left(P_{h}^{r} \otimes Q_{p}\right) u\right\|_{2} \\
& \leq\left\|u-\left(I \otimes Q_{p}\right) u\right\|_{2}+\left\|\left(I \otimes Q_{p}\right) u-\left(P_{h}^{r} \otimes Q_{p}\right) u\right\|_{2} \\
& =O\left(h^{r}+p^{-k}\right) .
\end{aligned}
$$


hp-version in the $x-y$ surface variables: Now we incorporate the $h p$-version of approximation technique in the $x-y$ coordinates. The goal is to approximate a function $u \in H^{k}(\omega \times \Gamma)$ from the tensor product space $S^{p_{1}, k}\left(\omega, T_{h}\right) \otimes P_{p_{2}}(\Gamma)$ for nonnegative integers $p_{1}$ and $p_{2}$. Analysis is similar to the one given in Lemmas 2.3 and 2.4 and therefore is not given. Using Theorem 2.2 , it can be seen easily that

Theorem 2.6 Let $u \in H^{k}(\omega \times \Gamma)$. Then there exists $u^{*} \in S^{p_{1}, k}\left(\omega, T_{h}\right) \otimes P_{p_{2}}(\Gamma)$,

$$
\left\|u-u^{*}\right\|_{L_{2}(\omega \times \Gamma)}=O\left(h^{\nu} p_{1}^{-k}+p_{2}^{-k}\right)
$$

where $\nu=\min \left(k, p_{1}+1\right)$ and $h=\max _{K \in T_{h}} \operatorname{diam}(K)$, with $T_{h}$ a triangulation of $\omega$.

Remark: Let $N(p) \equiv \frac{(p+1)(p+2)}{2}$. Note that numbers of the degrees of freedom of $S^{p_{1}, k}\left(\omega, T_{h}\right)$ and $P_{p_{2}}(\Gamma)$ are $M(h) N\left(p_{1}\right)$ and $N\left(p_{2}\right)$ respectively. Since a single element through the thickness is used because of the specific structural consideration in this paper, we can not expect the total error to decrease by letting the diameter $h \rightarrow 0$, -i.e., by letting the size of surface elements decrease to 0 . The second error term would quickly dominates the overall performance of approximation in that case. In order for both of the error terms in Theorem 2.6 to decrease consistently, note that $N(p)=O\left(p^{2}\right)$ and $h=O\left(M(h)^{-1}\right)$. Thus the number of surface elements $M(h)$ and the corresponding degree $p_{1}$ of polynomials should be selected so as to maintain

$$
M(h)^{-k} N\left(p_{1}\right)^{-\frac{k}{2}} \simeq N\left(p_{2}\right)^{-\frac{k}{2}}
$$

Equation (2.10) not only describes the consistent error estimates between the two terms but also indicates the consistent workloads between the surface and the through the thickness approximations. 


\section{Discontinuous Galerkin Method}

In this section, the discontinuous Galerkin (DG) method for problem (1.1) is developed. The discontinuity is introduced in time, which allows computation to march forward in time. This, when compared with the standard continuous Galerkin method, presents an enormous saving in size of computation. The DG finite element method for parabolic partial differential equations was studied in a series of papers by Erikson, Johnson and Larsson, $[8,9,10,11,12]$. In these papers, the convergence in time of $h$-finite element DG method is established for solutions which are smooth. More specifically, when the solutions are approximated by polynomials of degree $r$, then the algebraic error estimate of $O\left(\Delta t^{r+1}\right)$ as $\Delta t \rightarrow 0$ is obtained. However, in many parabolic partial equations, solutions exhibit singularities at $t=0$ due to the initial conditions. In a recent paper [1], the present authors established a graded time discretization scheme that captures the transient solution to optimal precision. The graded time mesh is selected by assuming that $\left\|u_{t}\right\|_{2}$ is weakly singular. A similar study of the graded time meshes is reported recently by Schötzau and Schwab [16]. They derive a set of graded time partition points by considering an incompatibility between initial and boundary conditions. It is demonstrated in [1] that the time discretization based upon $\left\|u_{t}\right\|_{2}$ provides more relaxed distribution of partition points. The paper of Schötzau and Schwab goes on to describe the $p$-finite element in time and obtain an exponential convergence in spite of a singular transient phase of the solution. We will not discuss the $p$-finite element in time in this paper. It will be taken up in [2] in which the complete $p$-finite element for parabolic problems is discussed.

We begin by recalling several results from [2] that are pertinent to the present paper. The following conditions will be assumed. Recall from Section 2 that $\Omega=\omega \times \Gamma$. Let $(h, T, S)$ denote a finite element discretization satisfying

1. $h$ is a positive function in $C^{1}(\bar{\Omega})$ such that

$$
|\nabla h(x)| \leq M, \quad \text { for all } x \in \bar{\Omega} \text { and for some } M>0
$$

2. $T=\{K\}$ is a set of triangular subdomain of $\omega$ with each triangular element having 
diameter $h_{K}$ such that

$$
c_{1} h_{K}^{2} \leq \int_{K} d x \quad \text { for all } K \in T
$$

and associated with the function $h$ through

$$
c_{1} h_{K} \leq h(x) \leq c_{2} h_{K}, \quad \text { for all } x \in K, K \in T
$$

where $c_{1}>0, c_{2}>0$

3. $S$ is the set of all continuous functions on $\bar{\Omega}$ which are polynomials of order $r$ in $x=\left(x_{1}, x_{2}\right)$ on each $K \in T$ and vanish on $\partial \omega$ as well as which are polynomials of order $p$ in the $z$-variable in $\Gamma$.

For the discontinuous Galerkin method for (1.1), we partition $R^{+}$as $0=t_{0}<t_{1}<$ $\cdots<t_{n}<\cdots$ where we let $I_{n} \equiv\left(t_{n-1}, t_{n}\right]$ with $k_{n} \equiv t_{n}-t_{n-1}$. For each time interval, with $q$ a nonnegative integer, We let

$$
W_{h p}^{q} \equiv\left\{v: R^{+} \rightarrow V_{h p}:\left.v\right|_{I_{n}} \in P_{q}\left(I_{n}\right), n=1, \ldots N\right\}
$$

where

$$
V_{h p}=\left\{\begin{array}{l}
\text { the space of all functions } u^{*} \in S_{h}^{r}(\omega) \otimes P_{p}(\Gamma) \\
\text { or } u^{*} \in S^{p, k}\left(\omega, T_{h}\right) \otimes P_{p}(\Gamma) \text { such that } \\
h=\max _{K \in T_{h}} \operatorname{diam}(K) \text { where } T_{h} \text { is a triangulation of } \omega
\end{array}\right\}
$$

and

$$
P_{q}\left(I_{n}\right)=\left\{v(t)=\sum_{i=0}^{q} v_{i} t^{i}: v_{i} \in V_{h p}\right\}
$$

The discontinuous Galerkin method is defined as follows:

Find $U$ such that for $n=1,2, \cdots$, with $\Omega=\omega \otimes \Gamma,\left.U\right|_{\Omega \times I_{n}} \in W_{h p}^{q}$ and

$$
\int_{I_{n}}\left\{\left(U_{t}, v\right)+a(U, v)\right\} d t+\left([U]_{n-1}, v_{n-1}^{+}\right)=\int_{I_{n}}(f, v) d t \quad \text { for all } v \in W_{h p}^{q}
$$

where $[w]_{n}=w_{n}^{+}-w_{n}^{-}, w_{n}^{+(-)}=\lim _{s \rightarrow 0^{+(-)}} w\left(t_{n}+s\right), U_{0}^{-}=u_{0},(u, v)=\int_{\Omega} u(x) v(x) d x$ and $a(u, v)=(\nabla U, \nabla v)$. The smoothness of $\left\|u_{t}\right\|_{L_{2}(\Omega)}$ is subject to the initial condition 
as well as to the boundary condition. For example, if we take in (1.1), $u_{0}(x)=\pi-x$, $f(x, t) \equiv 0$ and $\Omega=(0, \pi)$, then the actual solution of the corresponding problem is given by

$$
u(x, t)=\sum_{j=1}^{\infty} u_{j}^{0} e^{-j^{2} t} \sin (j x)
$$

where

$$
\begin{aligned}
u_{j}^{0} & =\frac{2}{\pi} \int_{0}^{\pi}(\pi-x) \sin (j x) d x \\
& =\frac{2}{\pi}\left\{\frac{\pi}{j}-\frac{1}{j^{2}} \sin ^{2} j \pi\right\} \\
& =O\left(\frac{1}{j}\right) .
\end{aligned}
$$

In the following, $C$ 's denote generic constants whose values change as they appear. Now,

$$
\left\|u_{t}(t)\right\|_{2}^{2}=\left\|u_{t}(t)\right\|_{L_{2}(\Omega)}^{2}=\sum_{j=1}^{\infty} C j^{2} e^{-2 j^{2} t}=\frac{d}{d t} \sum_{j=1}^{\infty} C e^{-2 j^{2} t}
$$

The last equality in (3.2) is justified because of the uniform convergence of $\sum_{j=1}^{\infty} C j^{2} e^{-2 j^{2} t}$ with respect to $t$. Now using the fact that $\int_{0}^{\infty} e^{-x^{2}} d x<\infty$, a simple change of variables (say, $y=j \sqrt{2 t}$ ) will show that the last expression in (3.2) is $\frac{d}{d t} C t^{-1 / 2}$, which leads to

$$
\left\|u_{t}(t)\right\|_{2}=O\left(t^{-3 / 4}\right)
$$

A similar argument shows that if $u_{j}^{0}=O\left(\frac{1}{j^{2}}\right)$ for some initial value function $u_{0}(x)$, then $\left\|u_{t}(t)\right\|_{2}=O\left(t^{-1 / 4}\right)$. This case arises when $u_{0}(x)=\min (x, \pi-x)$ for $x \in(0, \pi)$. If $u_{j}^{0}$ decays faster than $j^{-2.5}$ as $j \rightarrow \infty$, then $\left\|u_{t}(t)\right\|_{2}$ will be bounded as $t \rightarrow 0$. An initial phase for small $t$ is the well known initial transient for parabolic problems. It is the case that the smoothness of the solutions of parabolic problems vary significantly in space and time with initial transients where highly oscillatory components of the solution are decaying rapidly. Therefore, in order for numerical methods for parabolic problems to be successful, it is imperative that the methods take a careful account of time and space discretization scheme so as to capture the transient solutions. An adaptive time step control scheme was established by Eriksson and Johnson in [9]. Time steps $k_{n}$ are defined 
by controlling the size of

$$
\min _{j \leq q+1} k_{n}^{j}\left\|u_{t}^{(j)}\right\|_{I_{n}}
$$

where $q$ is the order of spline used in time and $u_{t}^{(1)}=u_{t}, u_{t}^{(2)}=u_{t t}, u_{t}^{(3)}=\Delta u_{t t}$ and $\|w\|_{I_{n}}=\max _{t \in I_{n}}\|w(t)\|_{2}$. Note that the method of Eriksson and Johnson requires some estimates concerning $\left\|u_{t t}\right\|_{I_{n}}$ and $u_{t}^{(3)}=\Delta u_{t t}$ to achieve the second and the third order convergence in time. The approach given in [1] provides convergence of any order in time for the discontinuous Galerkin method by examining only the behavior of $\left\|u_{t}\right\|_{2}$.

For $0<\alpha<1$ and $q$ a nonnegative integer, define $Q \equiv \frac{q+1}{1-\alpha}$. For a positive integer $N$ and $T>0$, define

$$
t_{n}^{*}=\left(\frac{n}{N}\right)^{Q}, \quad n=0,1, \ldots, N
$$

and

$$
t_{n}=t_{n}^{*} T
$$

We let $I_{n}=\left(t_{n-1}, t_{n}\right], \quad n=1,2, \ldots, N$. Let $k_{n}$ denote the length of $I_{n}$ so that

$$
k_{n}=\left[\left(\frac{n}{N}\right)^{Q}-\left(\frac{n-1}{N}\right)^{Q}\right] T, \quad n=1,2, \ldots N .
$$

Note that

$$
k_{n} \leq Q\left[\frac{n}{N}\right]^{Q-1} \frac{1}{N} T \quad \text { by the mean value theorem, }
$$

hence

$$
k_{n} \leq C \frac{1}{N^{q+1}}
$$

where $C$ is a constant independent of $n$. The solution $u(x, t)$ of $(1.1)$ is then approximated in $t$ over each $I_{n}$ by a polynomial of degree $q$. For example, with $q=1$, let $I_{n}^{i} w$ denote the linear interpolatory projection of $w \in H_{0}^{2}$ in time onto $W_{h k}$, viz,

$$
I_{n}^{i} w(x, t)=\frac{t_{n}-t}{k_{n}} w\left(x, t_{n-1}\right)+\frac{t-t_{n-1}}{k_{n}} w\left(x, t_{n}\right), \quad \text { for each } t \in I_{n} .
$$

Note that $I_{n}^{i}$, considered as an operator defined on $H_{0}^{2}$ is bounded with respect to the norm $\|\cdot\|_{\infty, I_{n}}$ where

$$
\|w(t)\|_{\infty, I_{n}} \equiv \max _{t \in I_{n}}\|w(t)\|_{L_{\infty}(\Omega)}
$$


Since $\Omega$ is assumed to be of bounded domain, $I_{n}^{i}$ is bounded with respect to $\|\cdot\|_{I_{n}}$ also. As was the case with the $L_{2}$ projection, $I_{n}^{i}$ equals the identity on polynomials of degree $\leq 1$. Expanding $u(x, t)$ in Taylor series with respect to $t$ at $t_{n}$ to the first or to the second order, we obtain, respectively, for each $n=1,2, \ldots, N$,

$$
\left\|u-I_{n}^{i} u\right\|_{I_{n}} \leq \int_{I_{n}}\left\|u_{t}(s)\right\|_{2} d s .
$$

Lemma 3.1 Let $0<\alpha<1, q$ a nonnegative integer and $T>0$, we assume that $t_{n}$, $n=1, \ldots, N$ are defined by (3.3). Then

$$
\max _{n \leq N} \int_{I_{n}} s^{-\alpha} d s \leq C_{n} \frac{1}{N^{q+1}},
$$

where $C_{n}$ is a constant independent on $N$.

Lemma 3.2 Let $t_{n}$ and $k_{n}$ be defined by (3.3). Then

$$
\left(1+\log \frac{t_{n}}{k_{n}}\right)^{1 / 2} \leq \sqrt{2}, \quad \text { for } \operatorname{each} n=0,1, \ldots, N .
$$

Lemma 3.2 is used to guarantee the stability of the discontinuous Galerkin method. In the remainder of this paper, we illustrate the current 'modified ' $h p$-finite element method by assuming the $h$-version in the surface $x-y$ variables using the linear splines. Also we illustrate the cases for constant as well as linear degree in time approximation. Let $\left\{\left(x_{i}, y_{i}\right)\right\}_{i=1}^{M}$ is the set of nodal points which are the interior vertices of $K$ in $T_{h}$. Let $\varphi_{j}$ be the linear spline basis element defined by $\varphi_{j}\left(x_{i}, y_{i}\right)=\delta_{i j}$, for $i, j=1, \ldots M$. The superscript $l$ used in (2.2) will be dropped. For application of higher order spline basis, more nodal points are required over each $K$. The solution $u$ of (1.1) is approximated by $(t>0)$

$$
\begin{gathered}
u(x, y, z, t) \sim \sum_{j=1}^{M}\left\{u\left(x_{j}, y_{j},-\frac{\Delta}{2}, t\right)\left(\frac{1}{2}-\frac{z}{\Delta}\right)+u\left(x_{j}, y_{j}, \frac{\Delta}{2}, t\right)\left(\frac{z}{\Delta}-\frac{1}{2}\right)\right. \\
\left.+\sum_{i=3}^{p+1} a_{i}^{j}(t) \psi_{i}(z)\right\} \varphi_{j}(x, y) .
\end{gathered}
$$

Note that $u\left(x_{j}, y_{j}, \mp \frac{\Delta}{2}, 0\right)$, for $j=1, \ldots, M$ are known from the initial condition. Also, for $t>0$, the boundary values $u\left(x_{j}, y_{j}, \mp \frac{\Delta}{2}, t\right)$ are given. As $u(\bar{x}, t)=0$, for $\bar{x} \in \partial \Omega, t \in R^{+}$ in (1.1), (3.6) simplifies to

$$
u(x, y, z, t) \sim \sum_{j=1}^{M} \sum_{i=3}^{p+1} a_{i}^{j}(t) \psi_{i}(z) \varphi_{j}(x, y) .
$$


At each time level $t_{n}$, we approximate $u\left(x, y, z, t_{n}\right)=u(\bar{x}, t)$ by

$$
U^{n}=U^{n}(\bar{x})=U\left(\bar{x}, t_{n}\right)=\sum_{j=1}^{M} \sum_{i=3}^{p+1} a_{i}^{j}\left(t_{n}\right) \psi_{i}(z) \varphi_{j}(x, y), \quad n=0,1, \ldots, N
$$

To start the DG finite element method, we first require $a_{i}^{j}\left(t_{0}\right)$ and they are determined from $u_{0}(\bar{x})$. More specifically, for each $j=1, \ldots, M$, since $u_{0}\left(x_{j}, y_{j}, z, t_{0}\right) \sim U^{0}\left(x_{j}, y_{j}, z, t_{0}\right)=$ $\sum_{i=3}^{p+1} a_{i}^{j}\left(t_{0}\right) \psi_{i}(z), M(p-1)$ many $a_{i}^{j}\left(t_{0}\right)$ are found by solving

$$
\sum_{k=3}^{p+1} \int_{-\frac{\Delta}{2}}^{\frac{\Delta}{2}} \psi_{k}(z) \psi_{i}(z) d z \cdot a_{k}^{j}\left(t_{0}\right)=\int_{-\frac{\Delta}{2}}^{\frac{\Delta}{2}} u\left(x_{j}, y_{j}, z, 0\right) \psi_{i}(z) d z, \quad \text { for } i=3, \ldots, p+1 .
$$

Now, equation (3.1) can be formulated as follows:

For $n=1,2, \ldots, N$, given $U^{n-1,-}$, find $\left.U \equiv U\right|_{I_{n}} \in P_{q}\left(I_{n}\right)$ such that

$$
\int_{I_{n}}\left[\left(U_{t}, v\right)+a(U, v)\right] d t+\left(U^{n-1,+}, v^{n-1,+}\right)=\int_{I_{n}}(f, v) d t+\left(U^{n-1,-}, v^{n-1,+}\right)
$$

for all $v \in P_{q}\left(I_{n}\right)$ where $U^{0,-}=u_{0}$.

For a special case, consider $q=0$, -i.e., constant in time. As $U^{n}=U^{n,-}=U^{n-1,+}$ in this case, (3.8) reduces to

$$
\left(U^{n}-U^{n-1}, v\right)+k_{n} a\left(U^{n}, v\right)=\int_{I_{n}}(f, v) d t,
$$

for all $v \in P_{0}\left(I_{n}\right)$ and $n=1,2, \ldots N$. With (3.7), (3.9) becomes for each $n=1,2, \ldots$

$\sum_{j=1}^{M} \sum_{i=3}^{p+1} a_{i}^{j}\left(t_{n}\right)\left[\left(\psi_{i} \varphi_{j}, \psi_{\alpha} \varphi_{\beta}\right)-k_{n} a\left(\psi_{i} \varphi_{j}, \psi_{\alpha} \varphi_{\beta}\right)\right]=\sum_{j=1}^{M} \sum_{i=3}^{p+1} a_{i}^{j}\left(t_{n-1}\right)\left(\psi_{i} \varphi_{j}, \psi_{\alpha} \varphi_{\beta}\right)+\left(f, \psi_{\alpha} \varphi_{\beta}\right)$, for each $\alpha=3, \ldots, p+1 ; \beta=1, \ldots, M$.

For $q=1$, we let $\left.U\right|_{I_{n}}=\Phi_{n}(\bar{x})+\frac{t-t_{n-1}}{k_{n}} \Psi_{n}(\bar{x})$ where $\Phi_{n}=\sum_{j=1}^{M} \sum_{i=3}^{p+1} a_{i}^{\Phi, j}\left(t_{n}\right) \psi_{i}(z) \varphi_{j}(x, y)$ and $\Psi_{n}=\sum_{j=1}^{M} \sum_{i=3}^{p+1} a_{i}^{\Psi, j}\left(t_{n}\right) \psi_{i}(z) \varphi_{j}(x, y)$. As $U^{n-1,+}=\Phi_{n}$ and $U^{n-1,+}=\Phi_{n-1}+\Psi_{n-1}$, (3.8) becomes

$$
\begin{aligned}
\int_{I_{n}}\left[\frac{1}{k_{n}}\left(\bar{\Psi}_{n}, v\right)+\right. & \left.a\left(\Phi_{n}+\frac{t-t_{n-1}}{k_{n}} \Psi_{n}, v\right)\right] d t+\left(\Phi_{n}, v_{+}^{n-1}\right) \\
& =\int_{I_{n}}(f, v) d t+\left(\Phi_{n-1}+\Psi_{n-1}, v_{+}^{n-1}\right)
\end{aligned}
$$


for all $v \in P_{1}\left(I_{n}\right)$. By taking $v=\psi_{\alpha} \varphi_{\beta}$ and $\frac{t-t_{n}}{k_{n}} \psi_{\alpha} \varphi_{\beta}$, (3.10) reduces to the following linear equations for $2 M(p-1)$ unknowns $a_{i}^{\Phi, j}\left(t_{n}\right)$ and $a_{i}^{\Psi, j}\left(t_{n}\right)$ :

$$
\begin{gathered}
\sum_{j=1}^{M} \sum_{i=3}^{p+1} \quad a_{i}^{\Phi, j}\left(t_{n}\right)\left\{\left(\varphi_{j} \psi_{i}, \varphi_{\beta} \psi_{\alpha}\right)+k_{n} a\left(\varphi_{j} \psi_{i}, \varphi_{\beta} \psi_{\alpha}\right)\right\} \\
+\sum_{j=1}^{M} \sum_{i=3}^{p+1} a_{i}^{\Psi, j}\left(t_{n}\right)\left\{\left(\varphi_{j} \psi_{i}, \varphi_{\beta} \psi_{\alpha}\right)+k_{n} a\left(\varphi_{j} \psi_{i}, \varphi_{\beta} \psi_{\alpha}\right)\right\} \\
=\int_{I_{n}}\left(f(t), \varphi_{\beta} \psi_{\alpha}\right)+\sum_{j=1}^{M} \sum_{i=3}^{p+1}\left[a_{i}^{\Phi, j}\left(t_{n-1}\right)+a_{i}^{\Psi, j}\left(t_{n-1}\right)\right]\left(\varphi_{j} \psi_{i}, \varphi_{\beta} \psi_{\alpha}\right) \\
\alpha=3, \ldots, p+1 ; \beta=1, \ldots, M \\
\left.\sum_{j=1}^{M} \sum_{i=3}^{p+1} \quad a_{i}^{\Phi, j}\left(t_{n}\right) \frac{k_{n}}{2} a\left(\varphi_{j} \psi_{i}, \varphi_{\beta} \psi_{\alpha}\right)+k_{n} a\left(\varphi_{j} \psi_{i}, \varphi_{\beta} \psi_{\alpha}\right)\right\} \\
+\sum_{j=1}^{M} \sum_{i=3}^{p+1} a_{i}^{\Psi, j}\left(t_{n}\right)\left\{\frac{1}{2}\left(\varphi_{j} \psi_{i}, \varphi_{\beta} \psi_{\alpha}\right)+\frac{k_{n}}{3} a\left(\varphi_{j} \psi_{i}, \varphi_{\beta} \psi_{\alpha}\right)\right\} \\
=\frac{1}{k_{n}} \int_{I_{n}}\left(t-t_{n-1}\right)\left(f(t), \varphi_{\beta} \psi_{\alpha}\right) d t \\
\alpha=3, \ldots, p+1 ; \beta=1, \ldots, M
\end{gathered}
$$

The following theorem can be proved by minor modifications to the proof of theorem 1.1, [9] and by making use of Theorem 2.5. The present theorem is described for $\Omega=$ $\omega \otimes \Gamma \subseteq R^{3}$.

Theorem 3.3 Suppose that there is a constant $\gamma$ such that the time steps $k_{n}$ satisfy $k_{n} \leq \gamma k_{n+1}, n=1, \ldots, N-1$ and let $U_{n}$ denote the solution of (3.8) approximating $u$ at $t_{n}$. Here $u$ is approximated by a polynomial of degree $q \geq 0$ over each $I_{n}$ for $n=1, \ldots, N-1$, and $u(\cdot, \cdot, \cdot, t)$ is approximated by an element from $S_{h}^{\tau}(\omega) \otimes P_{p}(\Gamma)$ for each $t \in R^{+}$, where $\omega$ is a polygonal domain in $R^{2}$. Then there is a constant $C$ depending only on $\gamma$ and $a$ constant $\beta$, where $\rho_{K} \geq \beta h_{K}$ and $\rho_{K}$ is the diameter of the circle inscribed in $K$ for all $K \in T_{h}$, such that for $n=1,2, \ldots, N$,

$$
\left\|u\left(t_{n}\right)-U_{n}\right\|_{2} \leq C\left(1+\log \frac{t_{n}}{k_{n}}\right)^{1 / 2}\left\{\max _{m \leq n}\left\|u-I_{n}^{i} u\right\|_{I_{m}}+h^{2}\left\|D_{x y}^{2} u\right\|_{I_{n}}+p^{-k}\|u\|_{I_{n}, H^{k}(\Gamma)}\right\},
$$

where $\|w\|_{I_{n}, H^{k}(\Gamma)}=\max _{t \in I_{n}}\|w(t)\|_{H^{k}(\Gamma)}$ and $D_{x y}^{2}$ denotes the second order derivative with respect to $x$ and $y$ variables.

Lemma 3.2 guarantees that the current DG finite element method with the graded temporal meshes defined in (3.3) is a stable scheme. Also Lemma 3.1 provides a bound for 
the term $\max _{n \leq m}\left\|u-I_{n}^{i} u\right\|_{I_{m}}$ in (3.11) provided that $\left\|u_{t}\right\|_{2}=O\left(t^{-\alpha}\right)$ as $t \rightarrow 0$. Theorem 2.5 is used to control the last two error terms in Theorem 3.3. In summary, we obtain the following theorem which utilizes the traditional $h$-finite element in the surface variables.

Theorem 3.4 Suppose that $u \in H^{k}(\omega \times \Gamma)$ and $\left\|u_{t}\right\|_{2}=O\left(t^{-\alpha}\right)$ for $0<\alpha<1$ and that the time partition points $t_{n}$ are taken according to (3.3). Let $U_{n}$ denote the solution of (3.8) approximating $u$ at $t_{n}$. Also assume that $u$ is approximated in time variable by a polynomial of degree $q \geq 0$ over each $I_{n}$ for $n=1, \ldots, N-1$, and $u(\cdot, \cdot, \cdot, t)$ is approximated by an element from $S_{h}^{2}(\omega) \otimes P_{p}(\Gamma)$ for each $t \in R^{+}$, where $\omega$ is a polygonal domain in $R^{2}$. Then

$$
\left\|u\left(t_{n}\right)-U_{n}\right\|_{2}=O\left(N^{-(q+1)}+h^{2}+p^{-k}\right)
$$

If higher order $r>2$ splines are used in $S_{h}^{r}(\omega)$, then the second term in the error can be replaced by $h^{r}$ provided $\left\|D_{x y}^{r} u\right\|_{I_{n}}$ is bounded.

In the case of the $h p$-finite element approximation for the surface variables, Theorem 2.6 is now used to establish the following.

Theorem 3.5 Suppose that $u \in H^{k}(\omega \times \Gamma)$ and $\left\|u_{t}\right\|_{2}=O\left(t^{-\alpha}\right)$ for $0<\alpha<1$ and that the time partition points $t_{n}$ are taken according to (3.3). Let $U_{n}$ denote the solution of (3.8) approximating $u$ at $t_{n}$. Also assume that $u$ is approximated in time variable by a polynomial of degree $q \geq 0$ over each $I_{n}$ for $n=1, \ldots, N-1$, and $u(\cdot, \cdot, \cdot, t)$ is approximated by an element from $S^{p_{1}, k}\left(\omega, T_{h}\right) \otimes P_{p_{2}}(\Gamma)$ for each $t \in R^{+}$, where $\omega$ is a polygonal domain in $R^{2}$. Then

$$
\left\|u\left(t_{n}\right)-U_{n}\right\|_{2}=O\left(N^{-(q+1)}+h^{\nu} p_{1}^{-k}+p_{2}^{-k}\right)
$$

where $\nu=\min \left(k, p_{1}+1\right)$.

Numerical experiments of the presently proposed 'modified" $h-p$ finite element method for parabolic equations will be reported elsewhere in future. 


\section{References}

[1] Hideaki Kaneko, Kim S. Bey and Gene J. W. Hou,Discontinuous Galerkin finite element method for parabolic problems,(preprint- Nov. 2000, NASA)

[2] Hideaki Kaneko and Kim S. Bey, Error analysis for p-version discontinuous Galerkin method for heat transfer in built-up structures,in preparation.

[3] I. Babuška and T. Janik, The hp-version of the finite element method for parabolic equations, I: The p-version in time,Numer. Methods P.D.E., Vol. 5, (1989), pp. 363-399.

[4] I. Babuška and T. Janik, The hp-version of the finite element method for parabolic equations, II: The hp-version in time,Numer. Methods P.D.E., Vol. 6, (1990), pp. 343-369.

[5] I. Babuška, B. A. Szabo and I. N. Katz, The p-version of the finite element method,SIAM Jl. Num. Anal., Vol. 18, No.3, (1981), pp. 515-545.

[6] I. Babuška and M. Suri, The $p$ and hp versions of the finite element method, basic principles and properties, SIAM Review, Vol. 36, No. 4, (1994), pp. 578-632.

[7] Claes Johnson, Numerical Solution of Partial Differential Equations by the Finite Element Method,Cambridge University Press, (1994).

[8] Kenneth Eriksson and Claes Johnson, Error estimates and automatic time step control for nonlinear parabolic problems, I, SIAM Jl. Num. Anal., Vol. 24, No. 1, (1987), pp.12-23.

[9] Kenneth Eriksson and Claes Johnson, Adaptive finite element methods for parabolic problems I: a linear model problem, SIAM Jl. Num. Anal., Vol. 28, No. 1, (1991), pp. $43-77$. 
[10] Kenneth Eriksson and Claes Johnson, Adaptive finite element methods for parabolic problems II: Optimal error estimates in $L_{\infty} L_{2}$ and $L_{\infty} L_{\infty}$, SIAM Jl. Num. Anal., Vol. 32, (1995), pp. 706-740.

[11] Kenneth Eriksson and Claes Johnson, Adaptive finite element methods for parabolic problems IV: Nonlinear problems, SIAM Jl. Num. Anal., Vol. 32, (1995), pp. 1729 1749.

[12] Kenneth Eriksson and Claes Johnson, Adaptive finite element methods for parabolic problems V: Long-time integration, SIAM Jl. Num. Anal., Vol. 32, (1995), pp. 17501763.

[13] W. Gui and I. Babuška, The $h, p$ and hp versions of the finite element method in 1 dimension, Part I. The error analysis of the p-version,Numer. Math., Vol. 49, (1986), pp. 577-612. Part II. The error analysis of the $h$ and $h p$ versions, Numer. Math., Vol. 49, (1986), pp. 613-657. Part III. The adaptive hp version, Numer. Math., Vol. 49, (1986), pp. 659-683.

[14] Hideaki Kaneko and Yuesheng Xu, Superconvergence of the iterated Galerkin methods for Hammerstein equations, SIAM Jl. Num. Anal., Vol. 33, No.3, (1996), pp. 1048-1064.

[15] Ch. Schwab, p- and hp- Finite Element Methods, Numerical Mathematics and Scientific Computation, Oxford Science Publication, Oxford University Press, (1998).

[16] D. Schötzau and Ch. Schwab, Time discretization of parabolic problems by the hpversion of the discontinuous Galerkin finite element method, SIAM Jl. Num. Anal., Vol. 38, No. 3, (2000), pp. 837-875.

[17] Larry Schumaker, Spline Functions: Basic Theory,John Wiley \& Sons, NY (1981). 